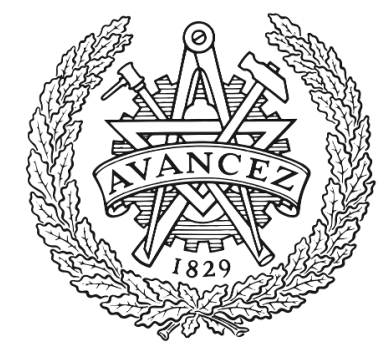

CHALMERS

UNIVERSITY OF TECHNOLOGY

\title{
An Integrated Ka-Band Diplexer-Antenna Array Module Based on Gap Waveguide Technology With Simple Mechanical Assembly and No
}

Downloaded from: https://research.chalmers.se, 2023-04-26 05:13 UTC

Citation for the original published paper (version of record):

Vosoogh, A., Sharifi Sorkherizi, M., Uz Zaman, A. et al (2018). An Integrated Ka-Band Diplexer-Antenna Array Module Based on Gap Waveguide Technology With

Simple Mechanical Assembly and No Electrical Contact Requirements. IEEE Transactions on

Microwave Theory and Techniques, 66(2): 962-972. http://dx.doi.org/10.1109/TMTT.2017.2757469

N.B. When citing this work, cite the original published paper.

(C2018 IEEE. Personal use of this material is permitted.

However, permission to reprint/republish this material for advertising or promotional purposes 


\title{
An Integrated Ka-Band Diplexer-Antenna Array Module Based on Gap Waveguide Technology with Simple Mechanical Assembly and no Electrical Contact Requirements
}

\author{
Abbas Vosoogh, Milad Sharifi Sorkherizi, Ashraf Uz Zaman, Jian Yang, and Ahmed A. Kishk, Fellow, IEEE,
}

\begin{abstract}
This paper presents the integration of a diplexer with a corporate feed network of a high gain slot array antenna at the Ka-band. A hybrid diplexer-splitter with a novel architecture is proposed to have compatibility for its direct integration with the feed-network of the array antenna. A $7^{\text {th }}$ order hybrid diplexer-splitter is successfully integrated into a corporate feed network of a $16 \times 16$ slot array antenna. The proposed integrated diplexer-antenna module consists of three distinct metal layers without the need of electrical contacts between the different layers based on the recently introduced gap waveguide technology. The designed module has two channels of $650 \mathrm{MHz}$ bandwidths each with center frequencies $28.21 \mathrm{GHz}$ and $29.21 \mathrm{GHz}$. The fabricated prototype provides good radiation and input impedance characteristics. The measured input reflection coefficients for both $T x / R x$ ports are better than $-13 \mathrm{~dB}$ with the measured antenna efficiency better than $60 \%$ in the designed passband, which includes the losses in the diplexer.
\end{abstract}

Index Terms-Diplexer, bandpass filter, group delay, gap waveguide, high efficiency, integration, millimeter-wave, slot array antenna, space mapping.

\section{INTRODUCTION}

A HIGH gain antenna connected to a diplexer is one integral part of any Frequency-division duplexing (FDD) communication link that has a major influence on the performance of the complete system. The integration of these two components can significantly reduce the total size and cost of the system and improves the system functionality. There have been some attempts to integrate antennas and diplexers [1][3]. However, in these examples, the proposed single antenna element does not provide enough gain for high data rate point-to-point wireless links, especially in the millimeter-wave frequency bands. Moreover, in all these cases, the diplexer is designed separately and connected to the antenna. Therefore, no major size reduction is observed in the total size of the module. The goal of this paper is to study the feasibility of integrating a practical diplexer into the feed-network of a large-scale array antenna in a compact form. Therefore, the

This work is financially supported by the Swedish Governmental Agency for Innovation Systems VINNOVA via a project within the VINN Excellence center Chase, and the European Research Council (ERC) under the 7th Framework Program ERC grant number 321125.

A. Vosoogh, A. Uz Zaman, and J. Yang are with the Electrical Engineering Department at Chalmers University of Technology, Gothenburg, Sweden (emails: \{abbas.vosoogh;zaman; jian.yang\}@ chalmers.se).

M. Sharifi Sorkherizi and A. A. Kishk are with the Electrical and Computer Engineering Department, Concordia University, Montreal, Canada. (e-mails: \{mi_shari; kishk\}@encs.concordia.ca). total size of the integrated module would be equal to that of the antenna.

Planar array antennas have several advantages over the reflector and lens antennas in terms of the size, assembly simplicity, and higher aperture efficiency. Therefore, they are preferred over the bulky lens and reflector antennas for many applications. Several works have been done to design highgain array antennas with improved efficiency. The microstrip and substrate integrated waveguide (SIW) have been used for this objective [4]-[9]. However, their associated losses are a major limiting factor for applications at high frequencies, such as at Ka-band and higher. Hollow waveguides, on the other hand, have the superior performance in terms of efficiency. However, ensuring the electrical contact between the different metal parts is an expensive and complicated procedure that limits the application of waveguide based high gain slot arrays especially at higher frequencies [10]. In order to achieve good electrical contact, diffusion bonding of thin copper plates is used in [11] and [12] to form corporate-feed hollow waveguide array antennas.

The gap waveguide technology that was proposed in [13] and [14] is an alternative to the conventional hollow waveguide to overcome the problem of the electrical contact between the different building blocks and still achieve low-loss performance, manufacturing flexibility, and cost effectiveness. Gap waveguide is based on parallel-plate waveguide configuration and using a semi-periodic array of electromagnetic bandgap structure to control the direction of propagation. By doing so, no electrical contact is required between the different metal blocks and metal layers. Several array antennas have been proposed in gap waveguide technology by using different fabrication techniques such as Computer Numerical Control (CNC) machining [15], [16], die-sink Electric Discharge Machining (EDM) [17], Direct Metal Laser Sintering (DMLS) 3D printing [18], and printed circuit board technology (PCB) [19], [20].

Design and optimization of diplexers is a topic that has been studied by many researchers for decades. Several geometries and methods have been proposed to calculate the lumped element model of the diplexers either by considering the impedance of the common port [21]-[28] or by optimization procedures [29], [30]. The major challenge is to translate the lumped element model to the physical dimensions of the structure since it involves numerical calculations of a 
computationally expensive structure due to its size. Generally, full-wave optimization and post fabrication tuning have major roles for this purpose. Bandpass filters are widely used to design diplexers [31], [32]. However, in some applications, where the relative bandwidth of the transmit and receive channels is large, it is better to use low-pass and high-pass filters combination instead of two bandpass filters [33].

The Q-factors of the ridge and groove gap waveguide resonators has been investigated in [34] and compared with $\mathrm{Q}$ of a conventional rectangular waveguide. It has been shown that groove gap and rectangular waveguide have similar $\mathrm{Q}$ values. Using gap waveguide technology, several prototypes of filters have been proposed, tested, and shown the effectiveness of this technology to realize low-loss filters, especially at higher frequency bands [35]-[41]. In [42] the performance of a groove gap waveguide filter has been compared to the same filter made in conventional rectangular waveguide technology with comparable performance. It has been shown that the electrical contact between the sidewalls and the upper plate is not needed in gap waveguide technology and does not affect the performance. However, in hollow waveguide components, good electrical and galvanic contact between the building blocks is critical. In [43] the integration of waveguide filters into a corporate-fed slot array antenna at E-band is presented. The authors have used diffusion bonding of several laminated thin copper plates to form the waveguide structure and maintain good electrical contact between the layers, which is an expensive process.

For the purpose of this paper, it is required to integrate a power combiner/divider into a diplexer in order to implement it in the middle of the feed-network of the array antenna in a way that each output port of the power combiner/divider excites each half of the feeding network equally. In [44] we presented a method to calculate the lumped element model of a hybrid diplexer-splitter using an efficient optimization routine. Here, the investigation is completed by providing more details on the design of the lumped model in Section III. Also, a method is proposed to translate the lumped model to physical dimensions efficiently without performing any full-wave optimization. In Section IV, the design of the $16 \times 16$ antenna array is presented. In Section $\mathrm{V}$ the designed hybrid diplexer-splitter and the array antenna are integrated to propose an efficient and novel module for Ka-band. Finally, experimental validation of the designed integrated diplexer-antenna array module is presented in Section VI.

\section{InTEGRATED DipleXER-ANTEnNA Module CONFIGURATION}

The configuration of the proposed integrated diplexerantenna module is shown in Fig. 1. The array antenna consists of three distinct metal layers with a small gap between each layer. The PEC/PMC stopband produced by the pins suppresses the undesired parallel-plate modes and leakage. The radiating layer consists of $16 \times 16$ slots with element spacing slightly smaller than one wavelength. The slots are fed by cavities on the middle layer. Each cavity feeds four slots in the form of a subarray to enable corporate-fed excitation of

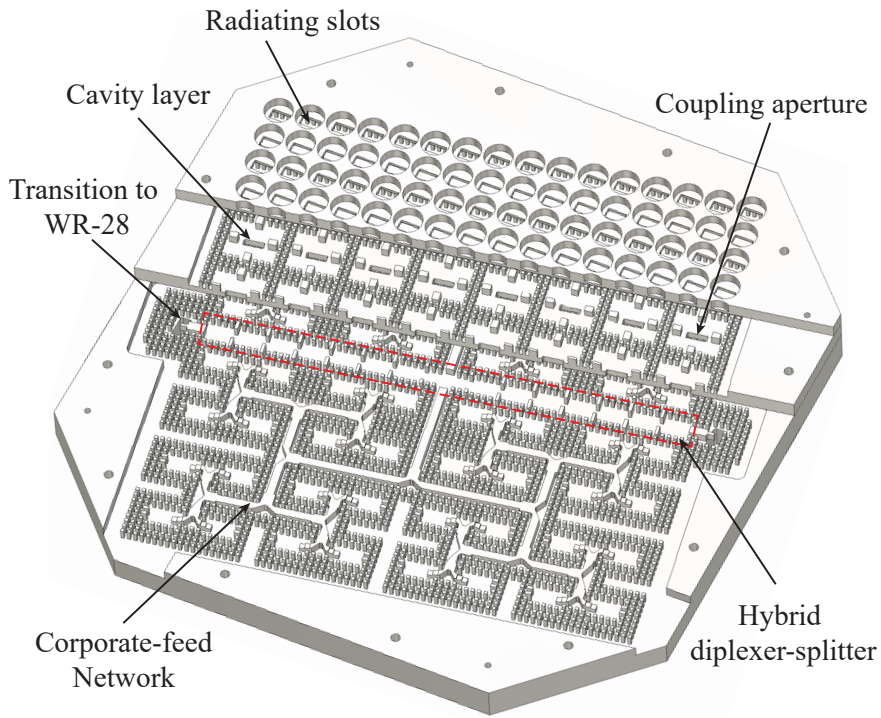

(a)

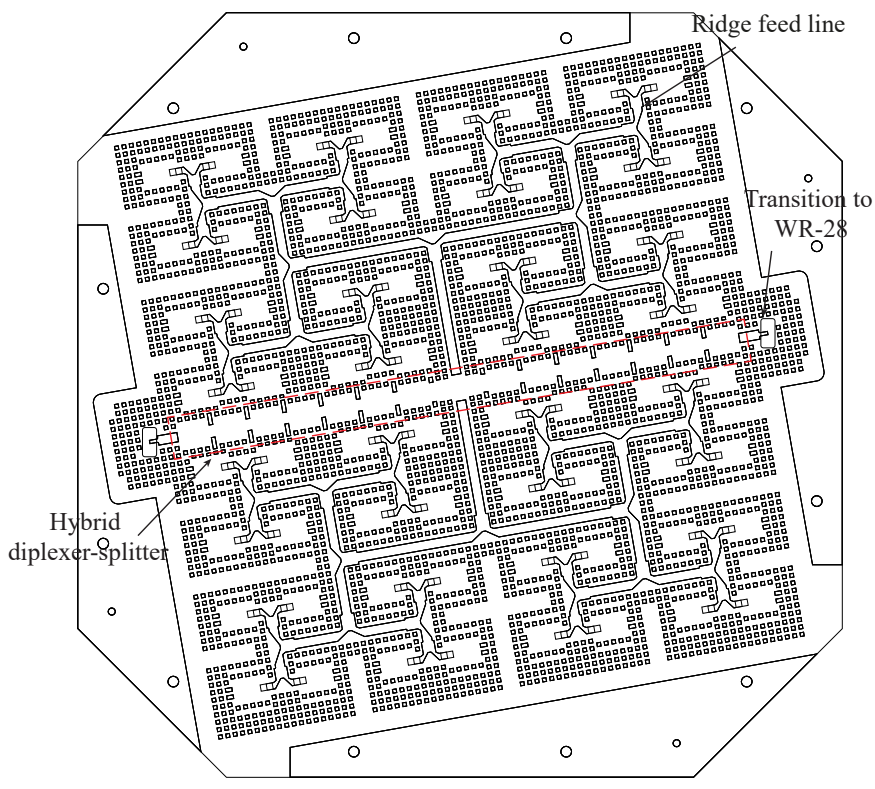

(b)

Fig. 1. (a) Configuration of the proposed integrated diplexer-antenna. (b) Distribution feed-network with an integrated diplexer.

slots, despite the limited space available to implement the feednetwork. In the lower layer, a ridge gap waveguide corporate feeding network is used to feed all the cavities via the coupling apertures.

The main element of the proposed module is a diplexer whose outputs are connected to a power combiner/divider. Fig. 1(b) shows the geometry of the distribution feed-network with integrated hybrid diplexer-splitter in more detail. The diplexer consists of two seventh-order bandpass filters, whose rectangular cavities and side walls are approximated by rows of pins. The designed hybrid diplexer-splitter is integrated into the feed-network of the array antenna in the middle. Each output of the hybrid diplexer-splitter feeds each half of the feed-network with the same phase and amplitude. A simple Eprobe transition is designed to match the ridge gap waveguide 
TABLE I

OPTIMIZED PARAMETERS OF THE LUMPED ELEMENT MODEL OF THE HYBRID DIPLEXER-SPLITTER (REFERS TO FIG. 2)

\begin{tabular}{lccc}
\hline \hline Parameter & value & Parameter & value $(\mathrm{GHz})$ \\
\hline$k_{0}$ & 0.0239 & $f_{0}$ & 28.6874 \\
$k_{1}$ & 0.0205 & $f_{1}$ & 28.1907 \\
$k_{2}$ & 0.0145 & $f_{2}$ & 28.1869 \\
$k_{3}$ & 0.0136 & $f_{3}$ & 28.1935 \\
$k_{4}$ & 0.0139 & $f_{4}$ & 28.2043 \\
$k_{5}$ & 0.0150 & $f_{5}$ & 28.2221 \\
$k_{6}$ & 0.0181 & $f_{6}$ & 28.2497 \\
$k_{7}$ & 0.0347 & $f_{7}$ & 28.3081 \\
$k_{8}$ & 0.0335 & $f_{8}$ & 29.1440 \\
$k_{9}$ & 0.0174 & $f_{9}$ & 29.1924 \\
$k_{10}$ & 0.0145 & $f_{10}$ & 29.2061 \\
$k_{11}$ & 0.0134 & $f_{11}$ & 29.2100 \\
$k_{12}$ & 0.0132 & $f_{12}$ & 29.2140 \\
$k_{13}$ & 0.0140 & $f_{13}$ & 29.2122 \\
$k_{14}$ & 0.0198 & $f_{14}$ & 29.2141 \\
$k_{15}$ & 0.0230 & & \\
$k_{16}$ & 0.0781 & & \\
\hline \hline
\end{tabular}

to a standard WR-28 rectangular waveguide at the back of the antenna. The proposed module is a compact design with a simple mechanical assembly without any requirement of electrical contact between the building blocks.

\section{HYBRID DIPLEXER-SPLITTER DESIGN}

The first step of the design is to calculate the lumped element model or the coupling matrix (CM) of a hybrid diplexer-splitter. To do so, we proposed a methodology in [44] that uses a modified goal function to calculate the CM of any integrated diplexer-power divider with Chebyshev responses. The coupling diagram of a hybrid seventh order channel diplexer-splitter is shown in Fig. 2. The diplexer is composed of two channels with seven resonators inter-coupled to each other. The diplexer also act as a power divider at the output. A new error function is presented in [44] in order to eliminate a spurious resonance between Port 3 and Port 4 through Resonator 0 . This has been done by applying a transmission zero in $S_{43}$ at the center frequency of the diplexer. The objective is to have channels with center frequencies at 28.21 $\mathrm{GHz}$ and $29.21 \mathrm{GHz}$ with $650 \mathrm{MHz}$ bandwidths.

The design procedure of the diplexer with $\mathrm{CM}$ in Fig. 2 is presented in [44]. In this section realization of the CM model using the delay response of sub-circuits is explained in more detail. Using the goal function in (1) of [44], the coupling values $(k)$ and resonant frequencies $(f)$ of the diagram of Fig. 2 are calculated and listed in Table I. For the output coupling of the diplexer (Port 3 and 4 to Resonator 0), the normalized coupling of $\mathrm{M}=1$ is used as the initial value. The normalization bandwidth is the total bandwidth of the diplexer (27.88-29.54 GHz). Furthermore, Resonator 0 is set to the center frequency of the diplexer.

The geometry of the realized CM is shown in Fig. 3. The structure consists of a semi-periodic array of metallic pins that together with the metal top plate create a bandgap to confine the energy inside the resonators without using any electrical contact between the lower part of the device and the top plate. Also, four guiding ridges are placed to work as the input/output of the device. Between each coupled cavity, a pair of extended pin controls the coupling. The value of the input/output couplings is controlled by the extension of the ridge into the resonators. The resonant frequency of the cavities is controlled by moving the semi-periodic pins away or close to each other. The dispersion diagram of the periodic metallic pin and the top plate unit cell is shown in Fig. 4, calculated by the CST Eigenmode solver. It is seen that the bandgap covers our band of interest.

Due to the relatively complex structure of gap waveguide components, the simulation time is generally high. Therefore, an efficient and fast procedure is needed in order to realize the $\mathrm{CM}$ of the structure in Fig. 3. We used the delay response of the sub-circuits and space mapping to calculate the cavity, iris, and ridge dimensions, without applying full-wave optimization. This method was introduced in [45], [46] for bandpass filters and diplexers. We have adapted this method with some modification for the proposed hybrid diplexers-splitter. The structure is divided into three sub-circuits. In each sub-circuit, few resonators are considered in the circuit, and the rest are de-tuned by metallic pins in their centers. Therefore, their resonant frequency is shifted to a higher frequency, but their physical influence is still considered in the EM simulation. The group delay response of $S_{11}, S_{22}$, and $S_{43}$ is used to calculate the CM parameters of each sub-circuit individually. Afterwards, the physical dimensions are calculated by using a linear mapping procedure. We used CST Microwave Studio for all simulations.

Fig. 5 shows Sub-circuit 1 in which the structure is excited at Port 3, and Resonators $0,5-7$ and 8-10 are in the circuits while the rest of them are detuned using shorting pins. To calculate the accurate phase information, a transition from the ridge waveguide to the ridge gap waveguide is used in the sub-circuit. This comes from the issues regarding defining a simulation port in CST for ridge gap waveguide. The effect of the transition will be removed by the procedure outlined in [45]. All the information about the resonant frequencies and couplings of the elements in Sub-circuit 1 can be extracted from the group delay of $S_{34}$. Fig. 6 shows the delay response of $S_{34}$ of Sub-circuit 1 after and before the phase removal procedure. As can be seen, this step is crucial to extract the correct couplings of the sub-circuit.

By performing an iterative space mapping procedure, all the cavity sizes and the iris dimensions are extracted. Also, the length of input ridges from Port 3, and Port 4 that extrudes to Cavity 0 is calculated. This length reflects the value of output coupling $(R O)$. The outcome of the optimization procedure is shown in Fig. 7 by presenting the compensated delay response of EM model and the response from the coupling matrix. The resonators at the end of channels (Resonator 5 and Resonator 10) are considered in the circuit in order to mimic the loading effect on their adjacent resonators, and their calculated dimensions will be disregarded later.

Sub-circuit 2 is shown in Fig. 8, which includes Resonators 1 to 6 while the rest of resonators are detuned. Group delay of reflection from Port 1 after phase removal is used to optimize the Sub-circuit. By optimizing Sub-circuit 2, dimensions of Resonators 1 to 5 and the iris dimensions incorporated with them will be calculated. It is important to note that Resonator 


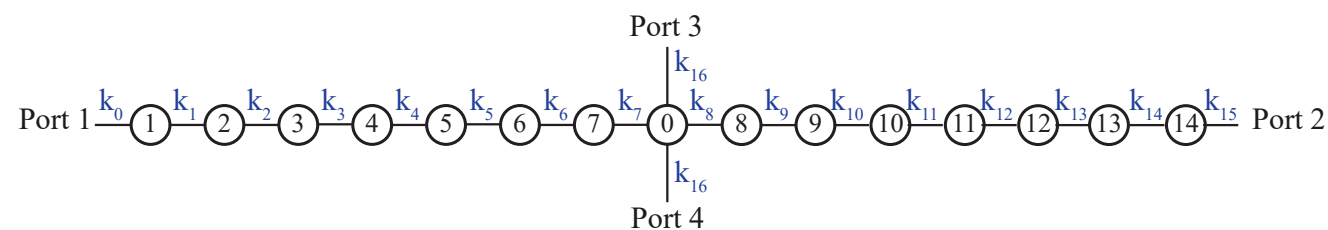

Fig. 2. Coupling diagram of a seventh order channel hybrid diplexer-splitter [44].

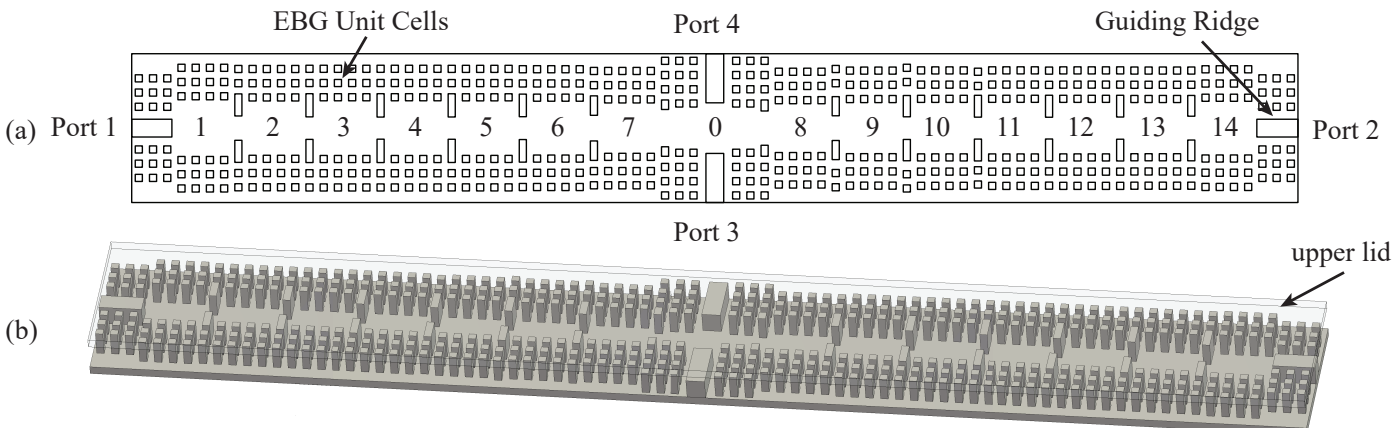

Fig. 3. Hybrid diplexer-splitter using gap waveguide technology. a) top view without the upper lid, b) 3D view.

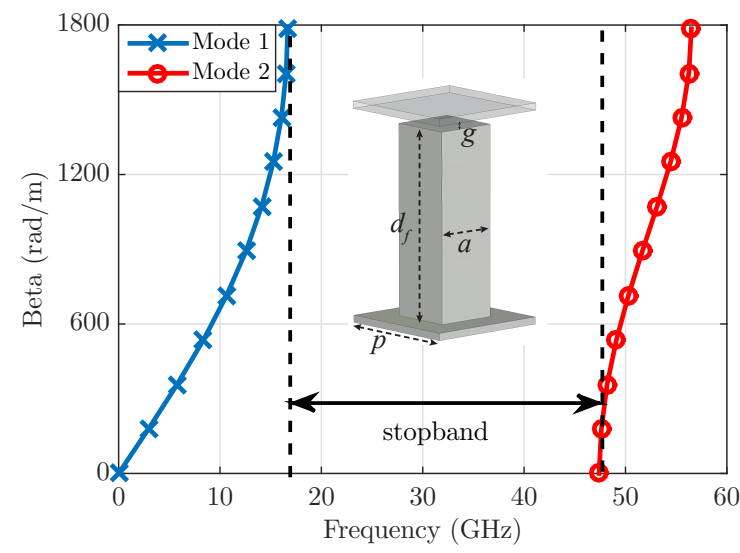

Fig. 4. Dispersion diagram and the size of the infinite periodic pin unit cell $\left(d_{f}=2.9 \mathrm{~mm}, a=0.9 \mathrm{~mm}, \mathrm{~g}=0.12 \mathrm{~mm}\right.$, and $\left.p=1.76 \mathrm{~mm}\right)$.

6 only exists in the circuit to mimic the loading effect on Resonator 5. The dimension for Resonator 6 calculated from Sub-circuit 2 should be replaced by the value from Sub-circuit 1. Also, the dimension calculated for resonator 5 is the correct value and should replace the value calculated from Sub-circuit 1. The outcome of the optimization of Sub-circuit 2 is shown in Fig. 9.

In a similar way, Sub-circuit 3 is generated as shown in

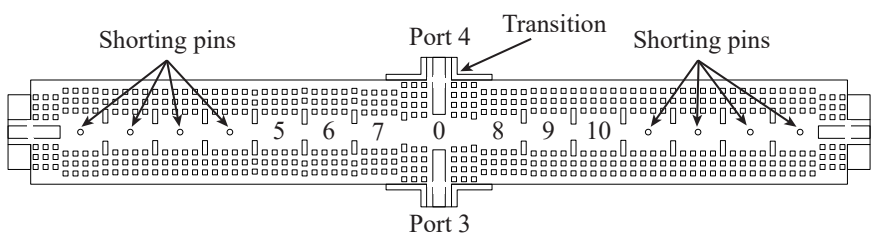

Fig. 5. Sub-circuit 1 of the hybrid diplexer-splitter.

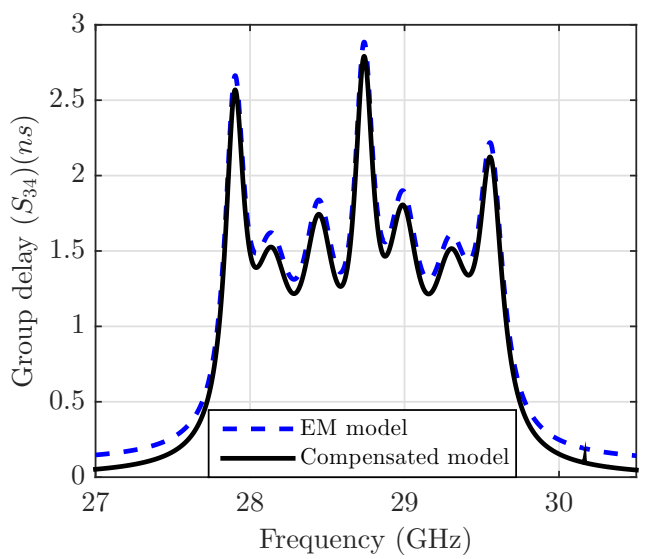

Fig. 6. Simulated delay response of Sub-circuit 1 after and before phase removal procedure.

Fig. 10. Resonators 9 to 14 are included in Sub-circuit 3. After performing the phase removal procedure and an iterative space mapping process, all the dimensions of the Sub-circuit 3 are calculated. The delay response of reflection from Port 2 for the optimized sub-circuits along with the expected response from the coupling matrix is plotted in Fig. 11, which shows the very close behavior. The dimension calculated for resonator 10 is the correct value and should replace the value calculated from Sub-circuit 1. If the outlined procedure is performed correctly, the iris dimension between Resonator 9 and 10 (responsible for coupling $k_{10}$ ) that is calculated from Sub-circuit 1 and also from Sub-circuit 3 should be equal or very close. This also applies to the iris between Resonator 5 and $6\left(k_{5}\right)$ that is calculated from Sub-circuit 1 and 2.

At this step, all the resonator widths, iris dimensions, and input/output lines are calculated based on the coupling diagram in Fig. 2. After removing all the shorting rods, the response 


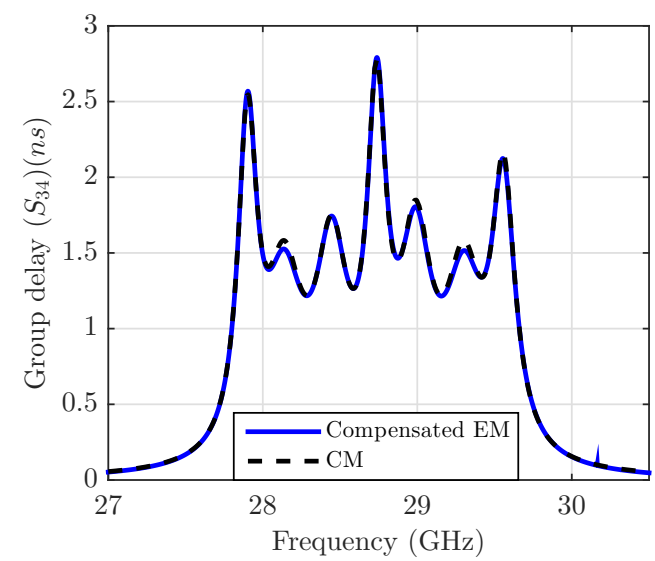

Fig. 7. Delay response of the optimized Sub-circuit 1 and the coupling matrix.

of the entire hybrid diplexer-splitter can be calculated. The optimized response of the design is shown in Fig. 12. The full-wave simulated results show an excellent agreement with the designed CM model. The focused transmission response is provided in Fig. 13. Ideally, at each output of the power divider, magnitude of $\left|S_{31}\right|,\left|S_{41}\right|,\left|S_{32}\right|$, and $\left|S_{42}\right|$ should be $-3 \mathrm{~dB}$ since the power is divided equally. However, due to the finite conductivity of the materials, a certain amount of loss is observed. Using Aluminum as the constructing material, a minimum of $0.9 \mathrm{~dB}$ insertion loss is noted at the outputs of both channels.

\section{Array Antenna Design}

Fig. 14 shows the configuration of $2 \times 2$ cavity-backed slot subarray, as the radiating element of the array antenna. Four slots with cylindrical cavities on the top of them are fed by an air-filled cavity, which is formed by the pins on the middle layer. The radiating element, i.e. slot layer and cavity layer are similar to the one in [47]. The slots are tilted by $10^{\circ}$ as explained in [47] in order to improve the far-field radiation characteristics of the antenna and satisfy the European Telecommunications Standards Institute (ETSI) class II sidelobe requirement. By this means, the sidelobe levels of the antenna are reduced by separating the E- and $\mathrm{H}$-planes of the antenna from the principal planes of the array.

In this paper, a new feed-network with a novel feeding mechanism is proposed for corporate-fed excitation of the cavities. In [47] T-shaped ridges are used to couple the quasiTEM mode of the feed ridge lines to the cavities via coupling slots. There is only one pin row separation between the ridge lines near the T-shaped ridges in the feed-network in [47].

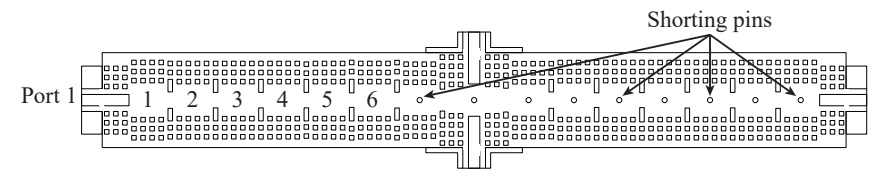

Fig. 8. Sub-circuit 2 of the hybrid diplexer-splitter.

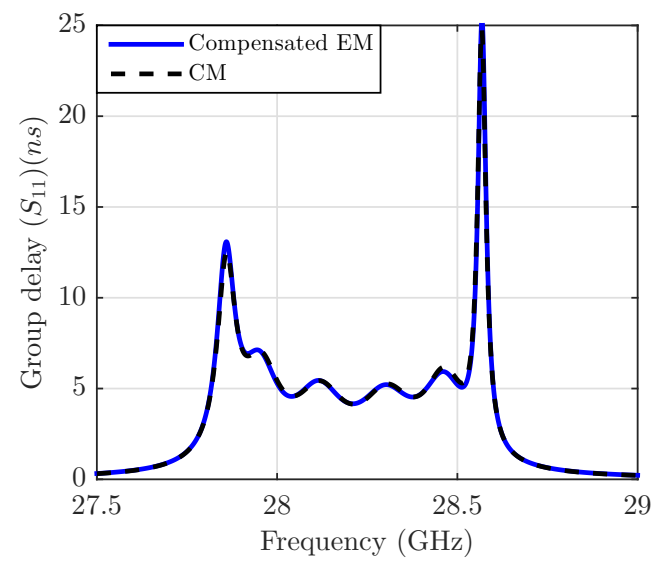

Fig. 9. Delay response of the optimized Sub-circuit 2 and the coupling matrix.

TABLE II

DIMENSIONS OF $2 \times 2$ CAVITY-BACKED SLOT SUBARRAY (REFERS TO FIG. 14 AND FIG. 15)

\begin{tabular}{lc}
\hline \multicolumn{1}{c}{ Parameter } & Value $(\mathrm{mm})$ \\
\hline Subarray width in the $\mathrm{x}$ and y-direction & 17.6 \\
Pins period $(p)$ & 1.76 \\
Width of the pins $(a)$ & 0.9 \\
Air gap $(\mathrm{g})$ & 0.12 \\
\hline \multicolumn{2}{c}{ Slot layer } \\
\hline Width of the slot $\left(w_{s}\right)$ & 3 \\
Length of the slot $\left(l_{s}\right)$ & 5.4 \\
Diameter of the cylindrical cavity $\left(D_{c}\right)$ & 8 \\
Height of the cylindrical cavity $\left(H_{c}\right)$ & 3 \\
\hline \multicolumn{2}{c}{ Cavity layer } \\
\hline Thickness of the cavity layer $\left(t_{c}\right)$ & 2.2 \\
Height of the cavity pins $\left(d_{c}\right)$ & 2.6 \\
Width of the coupling slot $\left(w_{c}\right)$ & 1.9 \\
Length of the coupling the slot $\left(l_{c}\right)$ & 5.9 \\
Length of the cavity $(l)$ & 6.6 \\
Width of the cavity $(w)$ & 5.8 \\
\hline \multicolumn{1}{c}{ Feeding layer } \\
\hline Width of the ridge $\left(w_{r}\right)$ & 1.5 \\
Height of the ridge $\left(d_{r}\right)$ & 2.4 \\
Height of the feeding pins $\left(d_{f}\right)$ & 2.9 \\
Length of the first matching step $\left(l_{m 1}\right)$ & 1.43 \\
Length of the second matching step $\left(l_{m 2}\right)$ & 1.45 \\
Height of the first matching step $\left(d_{m 1}\right)$ & 1.9 \\
Height of the second matching step $\left(d_{m 2}\right)$ & 1.37 \\
Width of the feeding cavity $\left(w_{f}\right)$ & 6.6 \\
Length of the feeding cavity $\left(l_{f}\right)$ & 6.7 \\
The coupling slot offset $\left(c_{f}\right)$ & 1.77 \\
Length of the matching pin $\left(a_{f}\right)$ & 1.56 \\
\hline \hline
\end{tabular}

Therefore, there is not enough space to place a diplexer in the middle of the feed-network for a compact integration.

Fig. 15 shows the feeding layer of the proposed subarray. The quasi-TEM mode of the ridge line is first coupled to

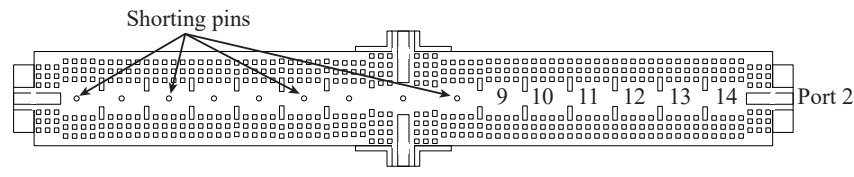

Fig. 10. Sub-circuit 3 of the hybrid diplexer-splitter. 


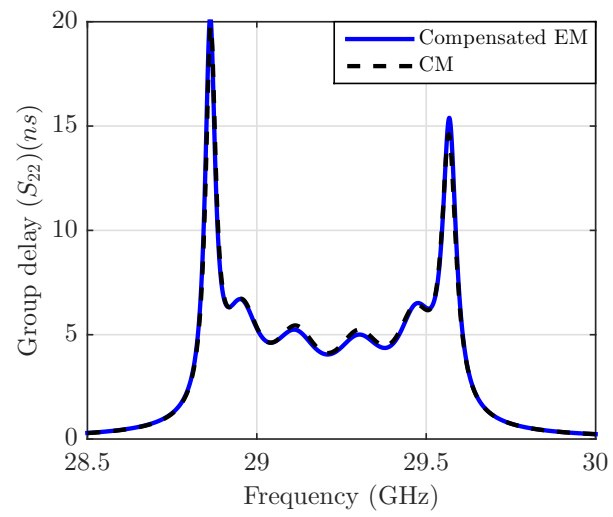

Fig. 11. Delay response of the optimized Sub-circuit 3 and the coupling matrix.

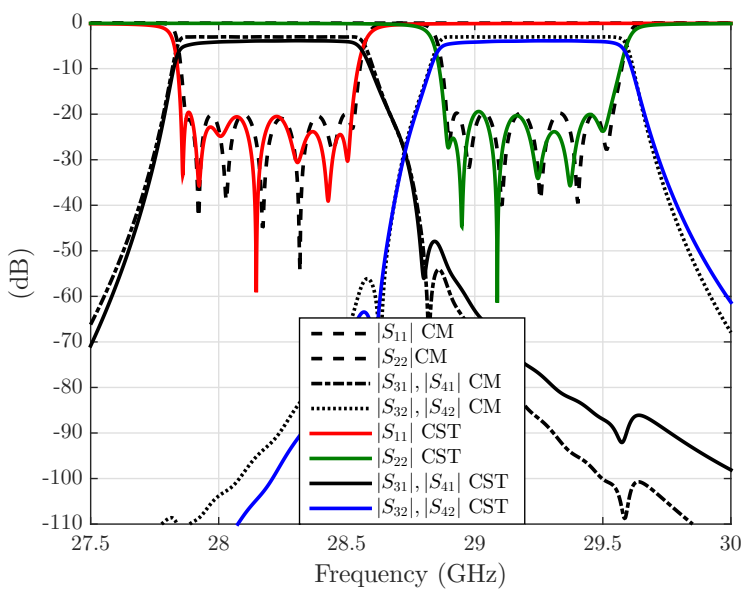

Fig. 12. Simulated EM response of the hybrid diplexer-splitter compared to CM.

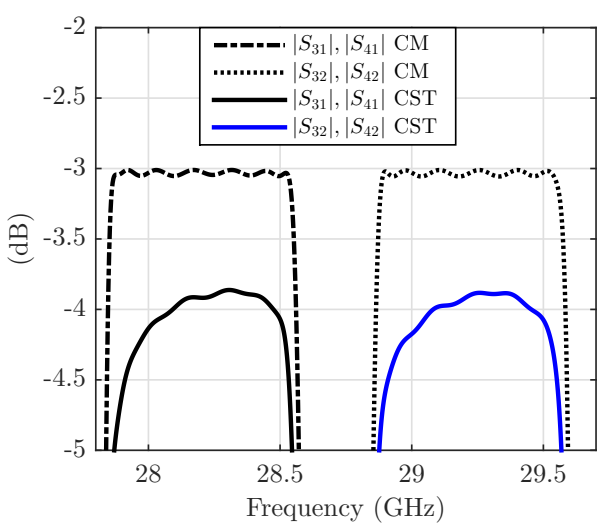

Fig. 13. Simulated transmission of the hybrid diplexer-splitter compared to CM.

a secondary cavity and then to the subarray via a coupling slot. With this new excitation, there are at least two pin rows between the ridge lines and consequently adequate space to integrate diplexer into the distributing feeding network of the array.

The proposed subarray is optimized using CST Microwave

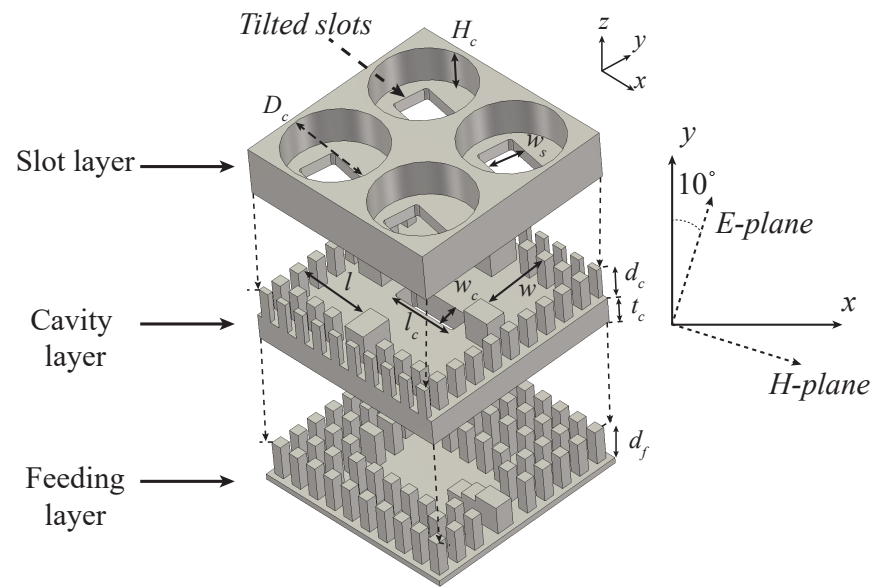

Fig. 14. Exploded view of $2 \times 2$ Cavity-backed slot subarray.

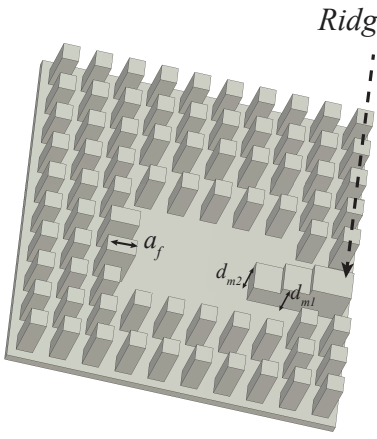

(a)

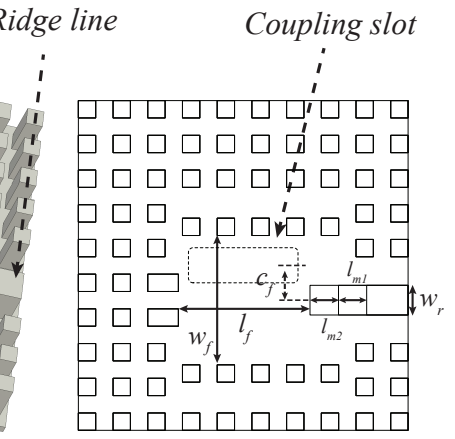

(b)
Fig. 15. The bottom feeding layer geometry.

Studio in infinite array environment with periodic boundary conditions. The simulated input reflection coefficient of the subarray is shown in Fig. 16. The final dimensions of the subarray are also presented in Table II. A full corporate feed network is designed by cascading E-plane ridge gap waveguide T-junctions with equal power division. The design procedure of the feed-network is similar to the one in [47]. The reflection coefficient of an $8 \times 8$ ways distribution network is also shown in Fig. 16. The output ports of the distributing network are terminated with waveguide ports in this figure. Furthermore, the simulated input reflection coefficient of the complete $16 \times$ 16 slot array antenna is also presented in Fig. 16. The response of the feed-network and full array are calculated using full wave simulations. The reflection coefficient of the whole array is below $-20 \mathrm{~dB}$ in the desired frequency band (over 27.9-29.9 $\mathrm{GHz})$.

Fig. 17 shows the geometry of a simple E-probe rectangular waveguide to ridge gap waveguide transition which is designed to match the ridge gap waveguide line to the standard WR-28 rectangular waveguide. To have an easier connection between the inputs of the antenna and the measurement equipment, the WR-28 opening is also rotated $10^{\circ}$ to be parallel to the radiating slots. The simulated S-parameters of the transition are shown in Fig. 18. The $S_{11}$ is below $-20 \mathrm{~dB}$ over the frequency band $27.5-31 \mathrm{GHz}$ and the simulated insertion loss 


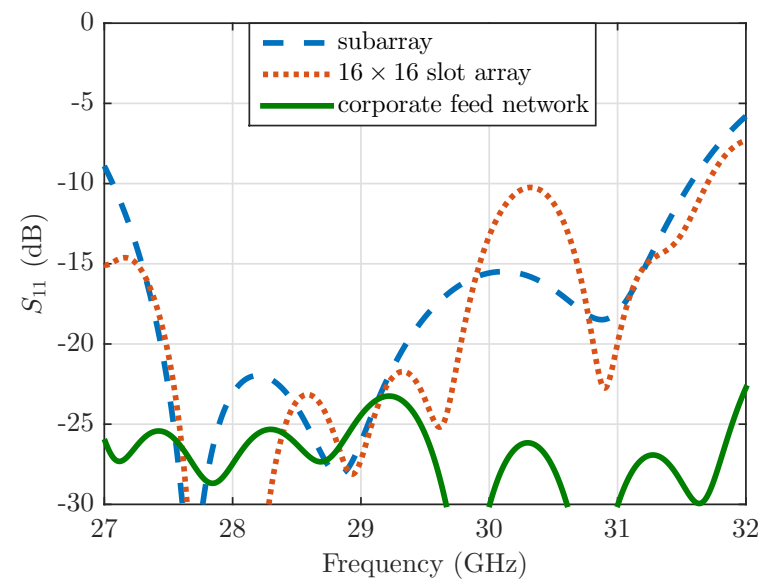

Fig. 16. Simulated reflected coefficient of the subarray, ridge gap waveguide feed-network, and complete $16 \times 16$ slot array.

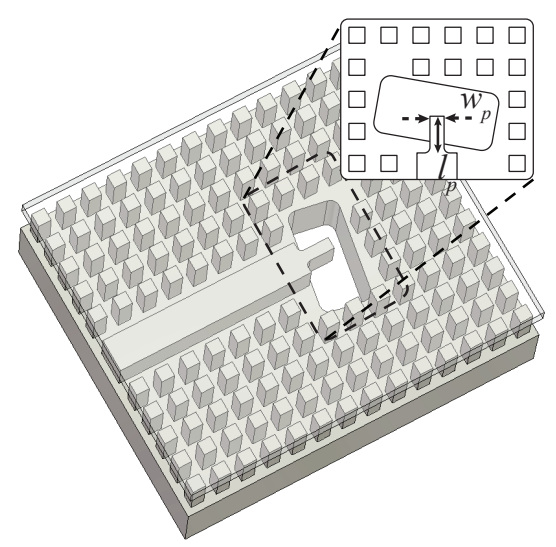

Fig. 17. Geometry of the rectangular waveguide to ridge gap waveguide transition $\left(w_{p}=0.78 \mathrm{~mm}\right.$, and $\left.l_{p}=2 \mathrm{~mm}\right)$.

is smaller than $0.06 \mathrm{~dB}$ with Aluminum as material over the same frequency band.

\section{INTEgRATION OF DiPLEXER AND THE ARRAY ANTENNA}

The designed hybrid diplexer-splitter is integrated into the distribution feed-network of the array antenna. Fig. 1(b) shows the feed-network of the antenna with the integrated diplexer. The outputs of the hybrid diplexer-splitter are connected to the feed-network at the middle of the antenna to act as the first power division stage. Therefore, the proposed architecture is very compact. The inputs of the diplexer are connected to WR-28 rectangular waveguides in the bottom plate via simple transitions.

The simulated time-averaged amplitudes of the E-field distribution within the feed-network of the antenna at the center frequencies of two Tx/Rx channels are illustrated in Fig. 19. As shown, despite the presence of a small gap between the layers and no electrical contact, there is no leakage between the feeding ridge lines and also between the diplexer and adjacent ridge lines. The latter is due to the stopband of the

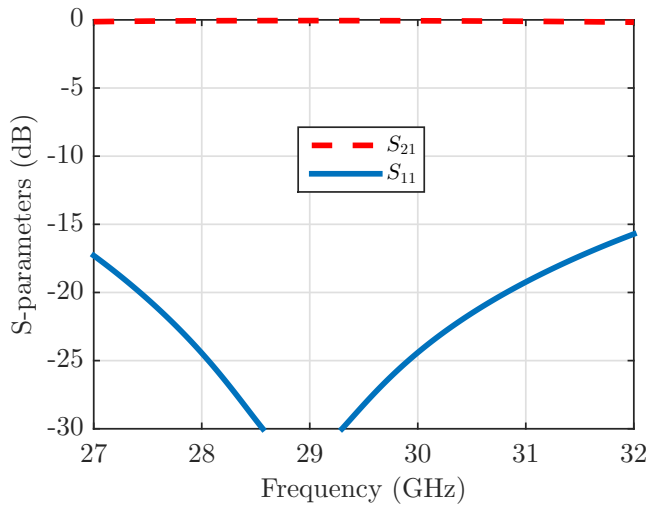

Fig. 18. Simulated performances of the rectangular waveguide to ridge gap waveguide transition.

pin texture, which prevents the propagation of the wave in the unwanted directions.

To investigate any possible leakage in the feed-network, the coupling between two parallel ridge lines with a different row of pins separation is studied. Coupling between port 1 and port 4 for two lines with a length of $30 \mathrm{~mm}$ is demonstrated in Fig. 20. The coupling increases by placing the ridge lines closer with fewer rows of pins separation. In the designed feeding network with an integrated diplexer, there are minimum two rows of pins between the ridge lines. The coupling between two parallel ridge lines is lower than -37 $\mathrm{dB}$ for two rows of pins separation and $-50 \mathrm{~dB}$ for three rows of pins separation. Therefore, coupling and leakage is not an issue in the designed gap waveguide feed-network.

A prototype consisting of $16 \times 16$ slots has been manufactured by $\mathrm{CNC}$ milling. The fabricated integrated diplexerantenna module is illustrated in Fig. 21. The fabricated antenna has a planar structure with an aperture size of $180 \times 180 \mathrm{~mm}^{2}$ and the total thickness of $10 \mathrm{~mm}$.

\section{EXPERIMENTAL RESULTS}

The measured and simulated input reflection characteristics of the manufactured module are presented in Fig. 22. There is a very good agreement between simulated and measured results. The measured reflection coefficients of the two Tx/Rx ports of the integrated diplexer-antenna module are below -13 $\mathrm{dB}$. There is almost no shift in frequency between the simulated and measured input reflection coefficients. The measured reflection coefficients are slightly higher than the simulated ones, which could be due to manufacturing and assembly tolerances. The measured isolation between two input ports is also illustrated in Fig. 23, which shows that there is no direct leakage between the two ports through the pins in the feed-network.

The simulated and measured gains of the fabricated module for two input ports are shown in Fig. 24. The simulated gain of the antenna without diplexer is also presented. The dashed lines in Fig. 24(b) correspond to the maximum available directivities for an aperture with the same size of the antenna with different aperture efficiencies. The simulated antenna efficiency without the diplexer is more than $85 \%$ over the 

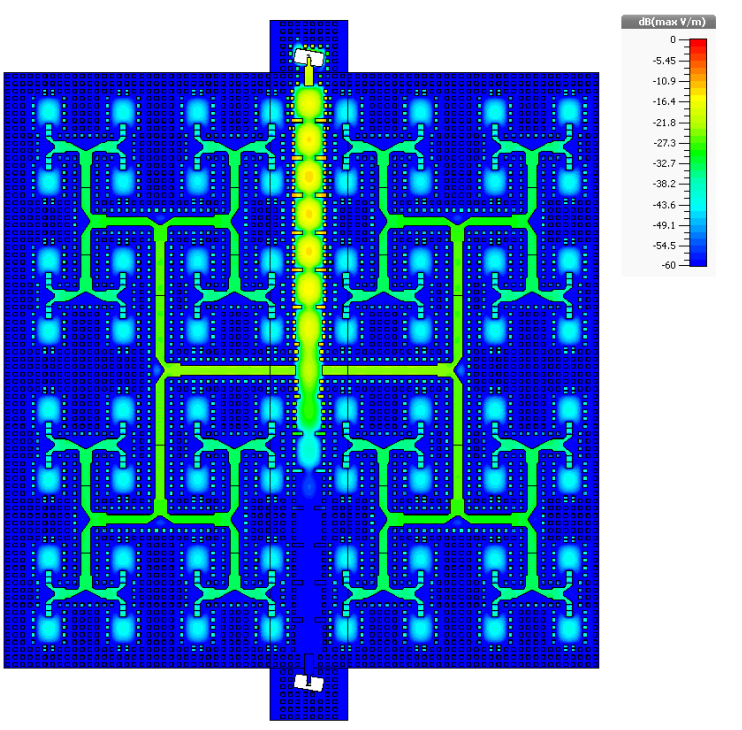

(a)
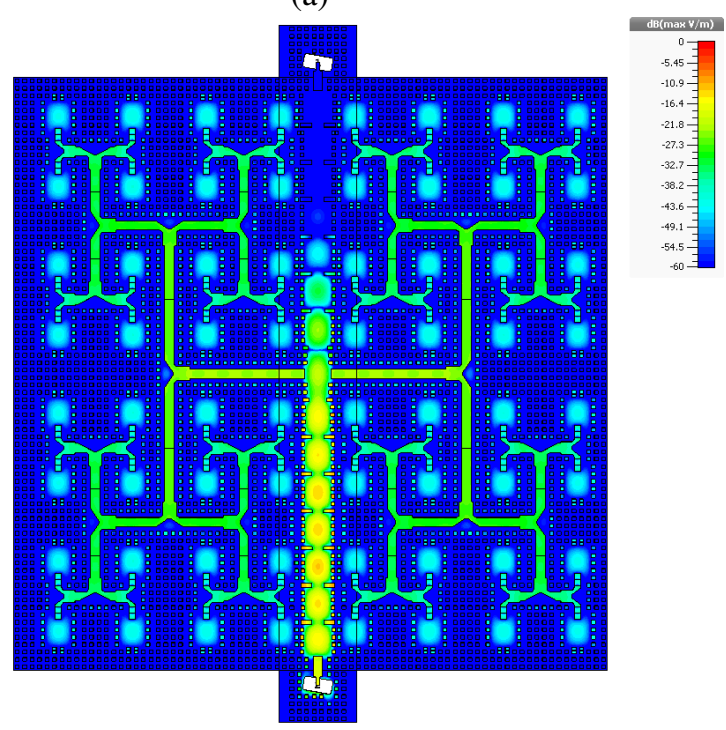

(b)

Fig. 19. Simulated E-field distribution within the feed-network. (a) $28.2 \mathrm{GHz}$, and (b) $29.2 \mathrm{GHz}$

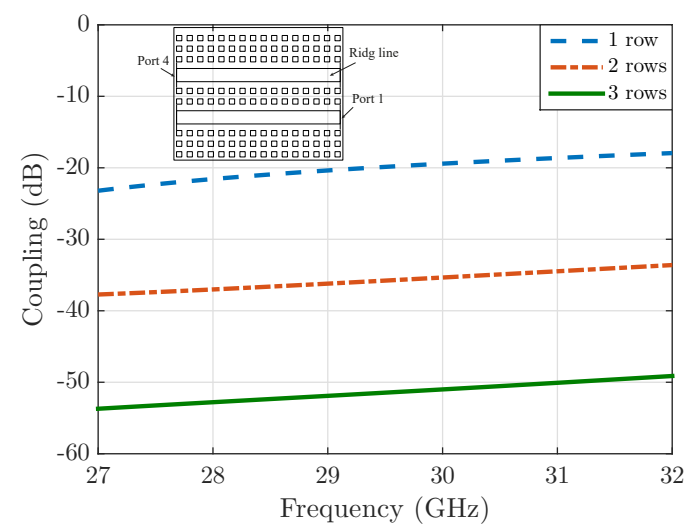

Fig. 20. Coupling between two parallel ridge lines separated by pin rows.

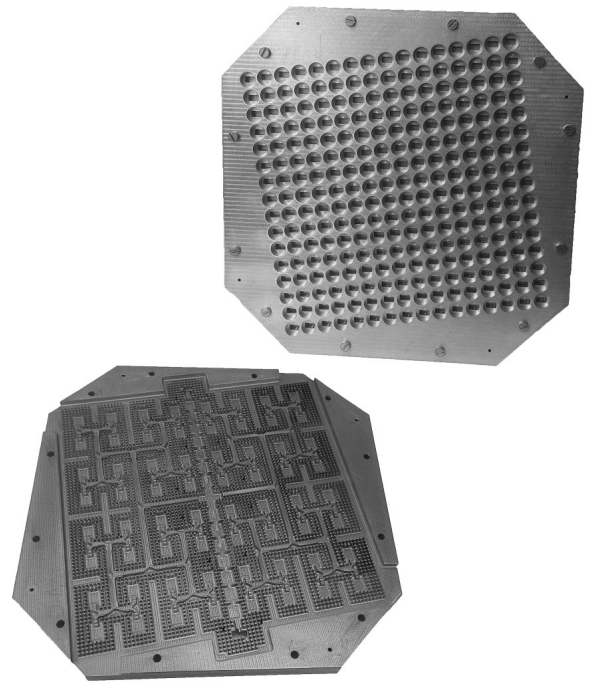

Fig. 21. Photograph of the fabricated integrated diplexer-antenna array module.

frequency band 27-30 GHz. The gain of the antenna is decreased after integrating the diplexer into the feeding network of the antenna. This drop is due to the losses introduced by the diplexer. The simulated efficiency of the antenna with diplexer is around 70\%. However, the measurements show lower gains and efficiencies. This reduction is attributed to the manufacturing tolerances and conductivity deterioration due to the surface roughness of the fabricated prototype. The simulated and measured gains of the two input ports of the proposed module are in good agreement with almost no shift in the operating frequency bands. The measured antenna efficiency of the fabricated integrated diplexer-antenna is around $60 \%$.

The normalized simulated and measured E- and H-planes radiation patterns of the antenna at the center of the two channels of the diplexer $28.2 \mathrm{GHz}$ and $29.2 \mathrm{GHz}$ are shown in Fig. 25 and Fig. 26, respectively. The designed antenna satisfies the ETSI class II Co-polar radiation pattern sidelobe requirement. The measured and simulated Co-polar radiation patterns are in good agreement, which shows that the hybrid diplexer-splitter feeds the two halves of the array feeding network with the same amplitude and phase. The measured cross-polar patterns are also presented in Fig. 25 and Fig. 26. The measured cross-polarization levels are better than $-35 \mathrm{~dB}$ in the both E- and H-planes.

\section{CONCLUSION}

The integration of a diplexer into a corporate feed network of a slot array antenna has been presented at the Ka-band. A compact integrated diplexer-antenna array has been designed using gap waveguide technology. The proposed module consists of three unconnected metal layers with simple mechanical assembly without the need of electrical contact between the metal blocks. This has been realized by using the stopband property of the pin texture to prevent any possible leakage and by controlling the direction of the wave propagation in a 


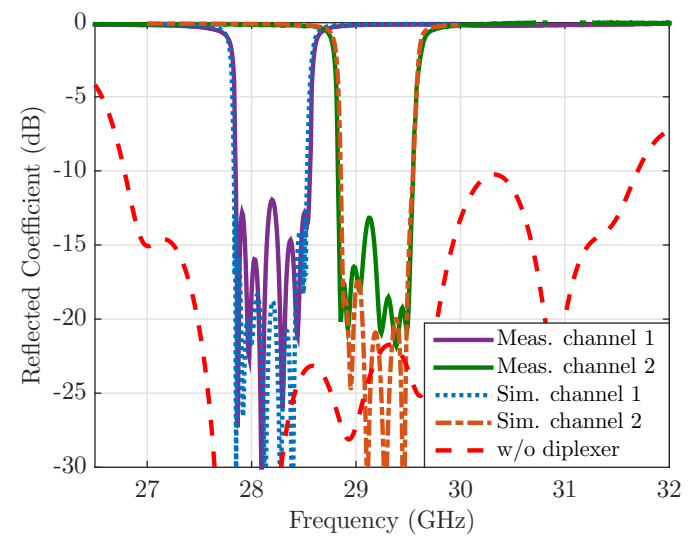

Fig. 22. Comparison of simulated and measured input reflection coefficients.

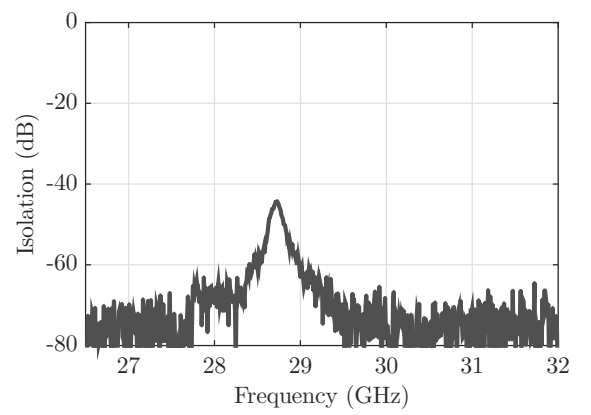

Fig. 23. Measured isolation between input ports.

parallel plate waveguide configuration. The detail design procedure of a new method for designing of large-scale filters and diplexers based on the group delay responses of sub-circuits in combination with space mapping has been presented. The proposed hybrid diplexer-splitter has been optimized with the proposed method efficiently with few full-wave simulations.

A prototype consisting of $16 \times 16$ cavity-backed slots with integrated diplexer has been manufactured with CNC milling. The fabricated prototype showed high gain and high efficiency. The designed integrated diplexer-antenna array has two channels with center frequencies at $28.21 \mathrm{GHz}$ and 29.21 $\mathrm{GHz}$ with $650 \mathrm{MHz}$ bandwidths, connected to standard WR28 rectangular waveguides at the back side of the antenna. The measured input reflection coefficients of both Tx/Rx ports are better than $-13 \mathrm{~dB}$. The proposed antenna has very good radiation characteristics and satisfies the ETSI class II Copolar sidelobe envelope requirement. The measured antenna efficiency is better than $60 \%$ in the designed passbands with high isolation of gain between two ports. The overall performance of the integrated diplexer-antenna module is quite promising, and this can open up new development of such integrated modules in the future.

\section{ACKNOWLEDGMENT}

This work had been done under the supervision of the late Prof. Per-Simon Kildal. The authors would like to express their gratitude to Prof. Per-Simon Kildal as he was a source of inspiration to the members of the antenna group at the

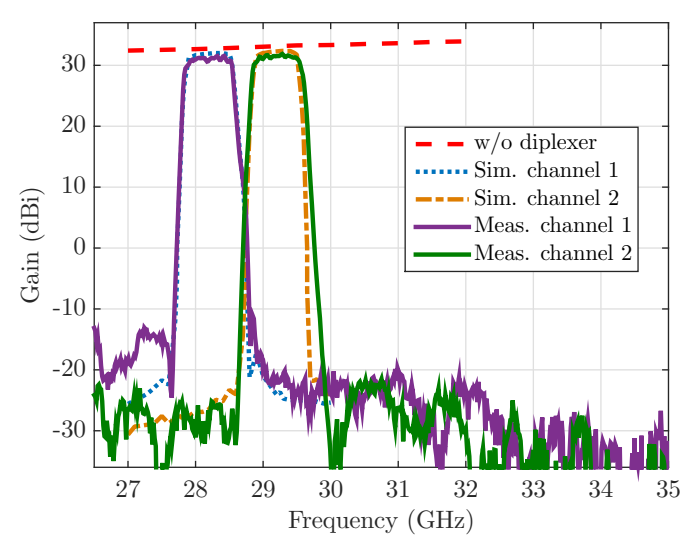

(a)

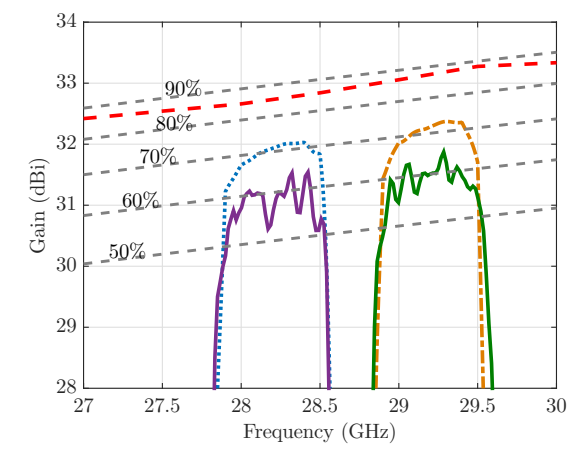

(b)

Fig. 24. Measured and computed gains of the designed integrated diplexerantenna module. (a) Overview, and (b) details in the channels. The dashed lines are the directivities with $90 \%, 80 \%, 70 \%$, and $60 \%$ aperture efficiencies.

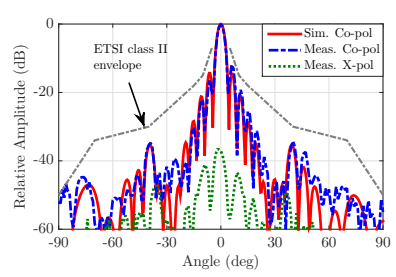

(a)

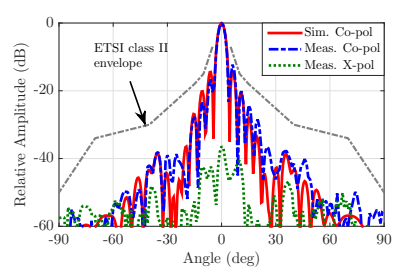

(b)
Fig. 25. Simulated and measured radiation patterns of the proposed array antenna at $28.2 \mathrm{GHz}$. (a) E-plane, and (b) H-plane.

Chalmers University of Technology. His absence will always be felt, and the work started by him on gap waveguide technology will be continued.

\section{REFERENCES}

[1] T.-Y. Yun, C. Wang, P. Zepeda, C. T. Rodenbeck, M. R. Coutant, M.-y. $\mathrm{Li}$, and $\mathrm{K}$. Chang, "A 10-to 21-GHz, low-cost, multifrequency, and fullduplex phased-array antenna system," IEEE Transactions on Antennas and Propagation, vol. 50, no. 5, pp. 641-650, May 2002.

[2] C. S. Cabello and E. Rajo-Iglesias, "Low cost self-diplexed antenna in inverted microstrip gap waveguide technology," in Antennas and Propagation (ISAP), 2014 International Symposium on, 2014, pp. 169170.

[3] Z. Kordiboroujeni, L. Locke, and J. Bornemann, "A diplexing antenna system in substrate integrated waveguide technology," in 2015 IEEE International Symposium on Antennas and Propagation \& USNC/URSI National Radio Science Meeting, 2015, pp. 1042-1043. 


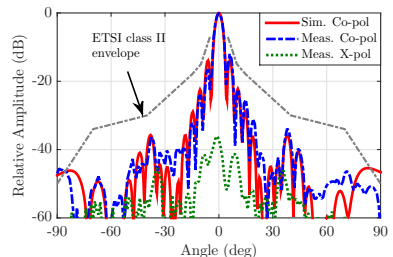

(a)

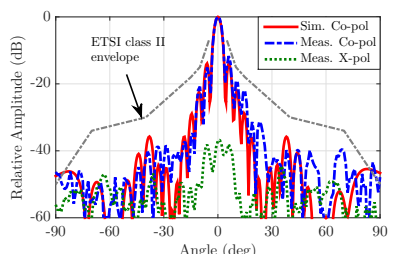

(b)
Fig. 26. Simulated and measured radiation patterns of the proposed array antenna at $29.2 \mathrm{GHz}$. (a) E-plane, and (b) H-plane.

[4] J. Wu, Y. J. Cheng, and Y. Fan, "A wideband high-gain high-efficiency hybrid integrated plate array antenna for V-band inter-satellite links," IEEE Transactions on Antennas and Propagation, vol. 63, no. 4, pp. 1225-1233, Apr. 2015.

[5] Y. J. Cheng, Y. X. Guo, and Z. G. Liu, "W-Band large-scale high-gain planar integrated antenna array," IEEE Transactions on Antennas and Propagation, vol. 62, no. 6, pp. 3370-3373, Jun. 2014.

[6] W. Yang, H. Wang, W. Che, Y. Huang, and J. Wang, "High-gain and low-loss millimeter-wave LTCC antenna array using artificial magnetic conductor structure," IEEE Transactions on Antennas and Propagation, vol. 63, no. 1, pp. 390-395, Jan. 2015.

[7] S. B. Yeap, Z. N. Chen, and X. Qing, "Gain-enhanced 60-GHz LTCC antenna array with open air cavities," IEEE Transactions on Antennas and Propagation, vol. 59, no. 9, pp. 3470-3473, Sep. 2011.

[8] J. Xu, Z. N. Chen, X. Qing, and W. Hong, "Bandwidth enhancement for a $60 \mathrm{GHz}$ substrate integrated waveguide fed cavity array antenna on LTCC," IEEE transactions on antennas and propagation, vol. 59, no. 3, pp. 826-832, Mar. 2011.

[9] Y. Li and K.-M. Luk, "60-GHz substrate integrated waveguide fed cavity-backed aperture-coupled microstrip patch antenna arrays," IEEE Transactions on Antennas and Propagation, vol. 63, no. 3, pp. 10751085, Mar. 2015.

[10] Y. Miura, J. Hirokawa, M. Ando, Y. Shibuya, and G. Yoshida, "Doublelayer full-corporate-feed hollow-waveguide slot array antenna in the 60GHz band," Antennas and Propagation, IEEE Transactions on, vol. 59, no. 8, pp. 2844-2851, Aug. 2011.

[11] K. Hashimoto, J. Takeuchi, J. Hirokawa, A. Hirata, and M. Ando, "Design and fabrication of a dual-polarization corporate-feed waveguide $32 \times 32$-slot array antenna for $120 \mathrm{GHz}$ band," in Antennas and Propagation (ISAP), 2014 International Symposium on. IEEE, 2014, pp. 33-34.

[12] Y. Miura, J. Hirokawa, M. Ando, K. Igarashi, and G. Yoshida, "A circularly-polarized aperture array antenna with a corporate-feed hollowwaveguide circuit in the $60 \mathrm{GHz}-$ band," in Antennas and Propagation (APSURSI), 2011 IEEE International Symposium on. IEEE, 2011, pp. 3029-3032.

[13] P.-S. Kildal, "Artificially soft and hard surfaces in electromagnetics," Antennas and Propagation, IEEE Transactions on, vol. 38, no. 10, pp. $1537-1544,1990$.

[14] P.-S. Kildal, A. U. Zaman, E. Rajo-Iglesias, E. Alfonso, and A. ValeroNogueira, "Design and experimental verification of ridge gap waveguide in bed of nails for parallel-plate mode suppression," IET Microwaves, Antennas \& Propagation, vol. 5, no. 3, pp. 262-270, Feb. 2011.

[15] A. Vosoogh and P.-S. Kildal, "Corporate-fed planar $60 \mathrm{GHz}$ slot array made of three unconnected metal layers using AMC pin surface for the gap waveguide," IEEE Antennas and Wireless Propagation Letters, vol. 15, pp. 1935-1938, Dec. 2015.

[16] A. U. Zaman and P.-S. Kildal, "Wide-band slot antenna arrays with single-layer corporate-feed network in ridge gap waveguide technology," IEEE Transactions on Antennas and Propagation, vol. 62, no. 6, pp. 2992-3001, Jun 2014

[17] A. Vosoogh, P.-S. Kildal, and V. Vassilev, "A multi-layer gap waveguide array antenna suitable for manufactured by die-sink EDM," in 2016 10th European Conference on Antennas and Propagation (EuCAP). IEEE, 2016, pp. 1-4.

[18] A. Vosoogh, P.-S. Kildal, V. Vassilev, A. U. Zaman, and S. Carlsson, "E-band 3-D Metal Printed Wideband Planar Horn Array Antenna," in Antennas and Propagation (ISAP), 2016 International Symposium on, 2016.

[19] S. A. Razavi, P.-S. Kildal, L. Xiang, E. Alfonso Alos, and H. Chen, " $2 \times 2$-slot element for $60-\mathrm{GHz}$ planar array antenna realized on two doubled-sided pcbs using SIW cavity and EBG-type soft surface fed by microstrip-ridge gap waveguide," Antennas and Propagation, IEEE Transactions on, vol. 62, no. 9, pp. 4564-4573, Sep. 2014.

[20] H. Attia, M. S. Sorkherizi, and A. A. Kishk, "60 GHz slot antenna array based on ridge gap waveguide technology enhanced with dielectric superstrate," in 2015 9th European Conference on Antennas and Propagation (EuCAP). IEEE, 2015, pp. 1-4.

[21] F. M. Vanin, D. Schmitt, and R. Levy, "Dimensional synthesis for wideband waveguide filters and diplexers," IEEE transactions on microwave theory and techniques, vol. 52, no. 11, pp. 2488-2495, Nov. 2004.

[22] A. Garcia-Lamperez, M. Salazar-Palma, and T. K. Sarkar, "Analytical synthesis of microwave multiport networks," in Microwave Symposium Digest, 2004 IEEE MTT-S International, vol. 2. IEEE, 2004, pp. 455458.

[23] A. Morini, T. Rozzi, and M. Morelli, "New formulae for the initial design in the optimization of T-junction manifold multiplexers," in Microwave Symposium Digest, 1997., IEEE MTT-S International, vol. 2. IEEE, 1997, pp. 1025-1028.

[24] J. D. Rhodes and R. Levy, "A generalized multiplexer theory," IEEE Transactions on Microwave Theory and Techniques, vol. 27, no. 2, pp 99-111, 1979.

[25] K.-L. Wu and W. Meng, "A direct synthesis approach for microwave filters with a complex load and its application to direct diplexer design," IEEE transactions on microwave theory and techniques, vol. 55, no. 5, pp. 1010-1017, May 2007.

[26] H. Meng and K.-L. Wu, "Direct optimal synthesis of a microwave bandpass filter with general loading effect," IEEE Transactions on Microwave Theory and Techniques, vol. 61, no. 7, pp. 2566-2573, Jul. 2013.

[27] G. Macchiarella and S. Tamiazzo, "Generation of canonical forms for multiport filtering networks," in Microwave Symposium (IMS), 2014 IEEE MTT-S International. IEEE, 2014, pp. 1-3.

[28] U. Rosenberg and S. Amari, "New power distribution (combination) method with frequency selective properties," in Eur. Microw. Conf., 2012, pp. 1-34.

[29] P. Zhao and K.-L. Wu, "An iterative and analytical approach to optimal synthesis of a multiplexer with a star-junction," IEEE Transactions on Microwave Theory and Techniques, vol. 62, no. 12, pp. 3362-3369, Dec. 2014.

[30] S. Amari, "Synthesis of cross-coupled resonator filters using an analytical gradient-based optimization technique," IEEE Transactions on Microwave Theory and Techniques, vol. 48, no. 9, pp. 1559-1564, Sep. 2000.

[31] E. Ofli, R. Vahldieck, and S. Amari, "Novel E-plane filters and diplexers with elliptic response for millimeter-wave applications," IEEE Transactions on Microwave Theory and Techniques, vol. 53, no. 3, pp. 843-851, 2005.

[32] J. Rebollar, J. Montejo-Garai, and A. Ohoro, "Asymmetric H-plane Tjunction for broadband diplexer applications," in Antennas and Propagation Society International Symposium, 2000. IEEE, vol. 4. IEEE, 2000, pp. 2032-2035.

[33] F. Teberio, I. Arregui, P. Soto, M. A. Laso, V. E. Boria, and M. Guglielmi, "High-performance compact diplexers for $\mathrm{Ku} / \mathrm{K}$-band satellite applications," IEEE Transactions on Microwave Theory and Techniques, 2017.

[34] E. Pucci, A. U. Zaman, E. Rajo-Iglesias, P.-S. Kildal, and A. Kishk, "Study of Q-factors of ridge and groove gap waveguide resonators," IET Microwaves, Antennas \& Propagation, vol. 7, no. 11, pp. 900-908, 2013.

[35] E. A. Alós, A. U. Zaman, and P.-S. Kildal, "Ka-band gap waveguide coupled-resonator filter for radio link diplexer application," IEEE Transactions on Components, Packaging and Manufacturing Technology, vol. 3, no. 5, pp. 870-879, 2013.

[36] M. Rezaee, A. U. Zaman, and P.-S. Kildal, "V-band groove gap waveguide diplexer," in Antennas and Propagation (EuCAP), 2015 9th European Conference on. IEEE, 2015, pp. 1-4.

[37] M. S. Sorkherizi, A. Khaleghi, and P.-S. Kildal, "Direct-coupled cavity filter in ridge gap waveguide," IEEE Transactions on Components, Packaging and Manufacturing Technology, vol. 4, no. 3, pp. 490-495, Mar. 2014.

[38] M. Rezaee, A. U. Zaman, and P.-S. Kildal, "A groove gap waveguide iris filter for V-band application," in 2015 23rd Iranian Conference on Electrical Engineering. IEEE, 2015, pp. 462-465.

[39] M. S. Sorkherizi and A. A. Kishk, "Fully printed Gap waveguide with facilitated design properties," IEEE Microwave and Wireless Components Letters, vol. 26, no. 9, pp. 657-659, 2016. 
[40] A. Vosoogh, A. A. Brazález, and P.-S. Kildal, "A V-band inverted microstrip gap waveguide end-coupled bandpass filter," IEEE Microwave and Wireless Components Letters, vol. 26, no. 4, pp. 261-263, Apr. 2016.

[41] A. Berenguer, M. Baquero-Escudero, D. Sanchez-Escuderos, B. Bernardo-Clemente, and V. E. Boria-Esbert, "Low insertion loss 61 $\mathrm{GHz}$ narrow-band filter implemented with groove gap waveguides ," in Microwave Conference (EuMC), 2014 44th European. IEEE, 2014 pp. 191-194.

[42] M. S. Sorkherizi and A. A. Kishk, "Completely tuned coupled cavity filters in defected bed of nails cavity," IEEE Transactions on Components, Packaging and Manufacturing Technology, 2016.

[43] X. Xu, M. Zhang, J. Hirokawa, and M. Ando, "E-band plate-laminated waveguide filters and their integration into a corporate-feed slot array antenna with diffusion bonding technology," IEEE Transactions on Microwave Theory and Techniques, vol. 64, no. 11, pp. 3592-3603, 2016.

[44] M. S. Sorkherizi, A. Vosoogh, A. A. Kishk, and P.-S. Kildal, "Design of integrated diplexer-power divider," in Microwave Symposium (IMS), 2016 IEEE MTT-S International. IEEE, 2016, pp. 1-3.

[45] M. S. Sorkherizi, A. A. Kishk, and M. Saad, "High rejection stacked bandpass filter optimized by group delay response," in 2015 IEEE MTTS International Microwave Symposium, 2015, pp. 1-4.

[46] M. S. Sorkherizi and A. A. Kishk, "Use of Group Delay of SubCircuits in Optimization of Wideband Large-Scale Bandpass Filters and Diplexers," IEEE Transactions on Microwave Theory and Techniques, 2017.

[47] A. Vosoogh, P.-S. Kildal, and V. Vassilev, "Wideband and High-Gain Corporate-Fed Gap Waveguide Slot Array Antenna with ETSI Class II Radiation Pattern in V-band," IEEE Transactions on Antennas and Propagation, 2016.

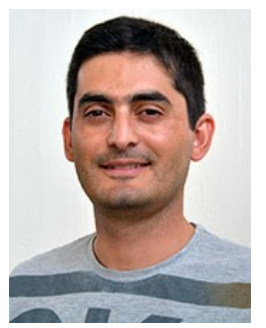

Abbas Vosoogh received the B.Sc. degree in electrical engineering from the University of Sistan and Baluchestan, Zahedan, Iran, and the M.Sc. degree from K. N. Toosi University of Technology, Tehran, Iran, in 2011 and 2018, respectively. He is currently pursuing his Ph.D. degree with Chalmers University of Technology, Gothenburg, Sweden. He revived the Best Student Paper Award at the 2015 International Symposium on Antennas and Propagation, Tasmania, Australis, the CST University Publication Award 2016, the Best Paper Award and the Best Student Paper Award at the 2016 International Symposium on Antennas and Propagation, Okinawa, Japan, and the First Prize Student Award at the 2017 IEEE International Symposium on Antennas and Propagation and USNCURSI Radio Science Meeting, San Diego, USA. His current research interests include the development of gap waveguide technology for millimeter and submillimeter wave applications, microwave passive components, EBG, soft and hard surfaces, mm-wave planar array antennas, and integration of passive and active components with array antenna.

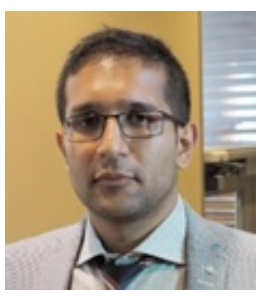

Milad Sharifi Sorkherizi received the B.Sc. and M.Sc. degrees in electrical and communication engineering from the K. N. Toosi University of Technology, Tehran, Iran, in 2010 and 2012, respectively, and the Ph.D. degree in electrical and computer engineering from Concordia University, Montreal, QC, Canada, in 2016. In 2016, he joined Apollo Microwaves Ltd., Dorval, QC, Canada, as a member of the Research and Development Team. His current research interests include microwave filters and diplexers, electromagnetic bandgap guiding structures, and millimeter-wave antenna arrays.

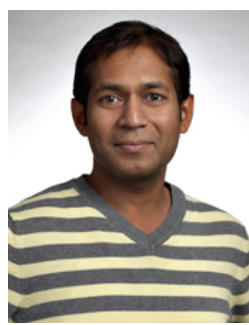

Ashraf Uz Zaman (M14) was born in Chittagong, Bangladesh. He received the B.Sc. degree in electri$\mathrm{cal}$ and electronics engineering from the Chittagong University of Engineering and Technology, Chittagong. He received the M.Sc. and PhD degree from Chalmers University of Technology, Gteborg, Sweden, in 2007 and 2013 respectively. He is currently an assistant professor with the Communication and Antenna Division of the same university. His current research interests include millimeter wave high efficiency planar antennas in general, gap waveguide technology, frequency selective surfaces, microwave passive components, packaging techniques and integration of MMICs with the antennas.

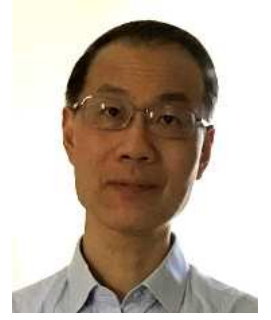

Jian Yang received the B.Sc. degree in electrical engineering from the Nanjing University of Science and Technology, Nanjing, China, in 1982, the M.Sc. degree in electrical engineering from the Nanjing Research Center of Electronic Engineering, Nanjing, in 1985, and the Swedish Licentiate and Ph.D. degrees from Chalmers University of Technology, Gothenburg, Sweden, in 1998 and 2001, respectively. From 1985 to 1996 , he was with the Nanjing Research Institute of Electronics Technology, Nanjing, China, as a Senior Engineer. From 1999 to 2005, he was with the Department of Electromagnetics, Chalmers University of Technology, Gothenburg, Sweden, as a Research Engineer. During 2005 and 2006, he was with COMHAT AB as a Senior Engineer. From 2006 to 2010, he was an Assistant Professor, from 2010 to 2016, he was an Associate Professor, and since 2016 he has been a professor with the Department of Signals and Systems, Chalmers University of Technology. His research interests include $60-140 \mathrm{GHz}$ antennas, terahertz antennas, MIMO antennas, ultrawideband (UWB) antennas and UWB feeds for reflector antennas, UWB radar systems,UWB antennas in near-field sensing applications, hat-fed antennas, reflector antennas, radome design, and computational electromagnetics.

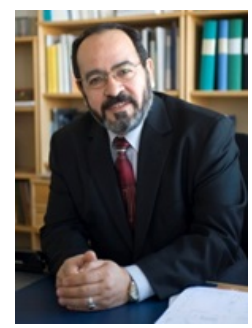

Ahmed A. Kishk received the BS degree in Electronic and Communication Engineering from Cairo University, Cairo, Egypt, in 1977, and BSc. in Applied Mathematics from Ain-Shams University, Cairo, Egypt, in 1980. In 1981, he joined the Department of Electrical Engineering, University of Manitoba, Winnipeg, Canada, where he obtained his M. Eng. and Ph.D. degrees in 1983 and 1986, respectively. Currently, he is a Professor at Concordia University, Montréal, Québec, Canada (since 2011) as Tier 1 Canada Research Chair in Advanced Antenna Systems. He was a distinguished lecturer for the Antennas and Propagation Society (2013-2015). He was an Editor of Antennas \& Propagation Magazine (1993-2014). He was an Editor-in-Chief of the ACES Journal from 1998 to 2001. He was a member of the AP AdCom (2013-2015). He is the 2017 AP president.

His research interest includes the areas of millimeter wave antennas for 5G applications, Analog beamforming network, Dielectric resonator antennas, microstrip antennas, small antennas, microwave sensors, RFID antennas for readers and tags, Multi-function antennas, microwave circuits, EBG, artificial magnetic conductors, soft and hard surfaces, phased array antennas, and computer aided design for antennas; reflect/transmitarray, wearable antennas, and Feeds for Parabolic reflectors. He has published over 300-refereed Journal articles and 450 conference papers. He is a co-author of four books and several book chapters and the editor of three books. He offered several short courses in international conferences. Dr. Kishk and his students received several awards. Dr. Kishk won the 1995 and 2006 outstanding paper awards for papers published in the Applied Computational Electromagnetic Society Journal. 